\title{
The Development and Aesthetic Pursuit of Opera Films in this Century (2000-2015)
}

\author{
Xiufeng Yang* \\ National Academy of Chinese Theatre Arts, Beijing 100073, China
}

\begin{abstract}
This paper examined the development of opera films in regard to cultural traditions and modernization, the evolution and analysis of new aesthetic concepts, the cultural reasons for continuous development and the expansion of communication channels. Based on these analyses, this paper concluded with the call for China to continuously improve and develop of the Chinese opera films in the hope for a new "golden age."
\end{abstract}

Keywords: Development; Opera films; Creation situation

Publication date: May, 2021; Publication online: 31 May, 2021

*Corresponding author: Xiufeng Yang, yxf55@sina.com

\section{Introduction}

With rapid development, various art forms are continuously enriched and developed. The internet has greatly impacted people's culture at an unimaginable speed. Each art form is a reflection to different trends along the timeline. After experiencing setbacks in the 1990's, the traditional Chinese opera films that progressed from stage to screen also followed the development in this century in terms of production, form of work, and aesthetic performance, thus, indicates a flourishing progress.

\section{Development in Cultural Traditions and Keeping Them Alive}

In 2001, Kunqu Opera ranked first among the first batch of 19 "Masterpieces of the Oral and Intangible Heritage of Humanity" announced by UNESCO. Kunqu Opera is recognized and appreciated worldwide for its unique artistic and cultural values, which has motivated the entire Kunqu Opera and the opera circle.

The subsequent introduction of a series of wholesome "plans", "methods" and "procedures" has established the status of "opera" in the development of national culture. Having such heritage has consolidated the foundation of innovation for future developments in the cultural industry. The affluence of the opera stage naturally led to the creation of opera films. Hence, it is worth paying attention to the following aspects:

\subsection{Remake of classic Chinese opera films as spectacular innovations}

In the production of opera films for more than a hundred years, many films have demonstrated their unique artistic charms in terms of selection of repertoires, actor's performances, and film presentations. Many of these films have become classics such as "The Wild Boar Forest," "The Female Generals of the Yang Family," which is directed by Cui Wei and Chen Huaiai, "Fifteen Strings of Coppers," directed by Tao Jin, "Dream of the Red Chamber," directed by Cen Fan, "Liang Shanbo and Zhu Yingtai," directed by Sang Hu and Huang Sha, etc. They are all influential works from the 1950's and 1960's. There are common features among these productions. First, these are excellent original repertoires that have been performed on stage. Second, the actors in these productions are famous artists of various dramas, and thirdly, the artistic value in stage performance is creatively transformed in these films, whereby the unique charm of the opera is better demonstrated. 
The production of other opera films such as " $\mathrm{A}$ Dream of Red Mansions" (Kun Opera in 2007), "The Legend of the White Snake" (Peking Opera in 2007), and "Liang Shanbo and Zhu Yingtai" (Yue Opera in 2009) are spectacular innovations in this century.

"The Legend of White Snake" (Peking Opera) played by Zhang Huoding and Song Xiaochuan in 2007, “The Legend of White Snake" (Peking Opera) played by Li Ping and Song Xiaochuan in 2013, and "The Legend of White Snake"" played by Jin Jing and Ye Jing in 2015 (Yue Opera), have their own artistic charms, genres, character portrayals, and conveyance of emotions. In addition, the aesthetic appeal by the creative team also contributes to the film's style.

Since 2000, the traditional opera, "Dream of Red Mansions" has been made into traditional opera films for three times. In 2007, the Central Studio for Documentary Film launched the "Rescue Project of National Cultural Repertoires." "A Dream of Red Mansions" (Yue Opera) was the first project launched. It was based on the Yue Opera recorded by the Chinese record company in 1961 and the adaptation was produced by Director Cen Fan in 1962. In this project, the deleted songs in the 1962 adaptation were restored and it focused on expressing the emotions of Bao and Dai. Soon thereafter, an orchestral adaptation of "A Dream of Red Mansions" was released. Five years later, in 2012, the Northern Kunqu Opera Theater presented the same repertoire, however, this production not only tells of the love story of Bao-Dai, but also illustrates the rise and fall of the Rongguo Building from Baoyu's perspective. These three works have their own unique artistic charms in content and form. Classic works are inherited from generation to generation, and it would be a challenge to reproduce the same repertoire. They are continuously polished, refined and fine-tuned for an exceptional inheritance process. Generally, different types of productions of the same repertoire display their own styles and artistic charms.

\subsection{Focusing on new rural development}

Rural themes have a certain weight in the production of modern operas. Local operas with $\mathrm{Yu}$, Huagu, and Ping opera as their inspirations have more advantage in portraying rural themes, in addition to more freedom in terms of performance. The lively language used and performance such as singing, dancing, etc., displays a prosperous element. Changes in people's living conditions and ideology in social development with new rural development have become interests for creative contents in this period.

The shadow puppet show, "The Road to a Well-off Society" is about a town chief who leads masses to overcome difficulties and improve economically. On the other hand, the Hunan flower drum opera's "The head of the township is Zhao," humorously depicts how cadres do practical things for the masses and the Yu opera's "Country Police Officer" which is based on real characters is about the relationship between cadres and the masses. Huangmei Opera's "Song Nostalgia" reflects young people's involvement in advancing the agricultural sector with science and technology, meanwhile, Yu Opera's "Village Officer Li Tiancheng" tells of the villager's diligent work in order to be wealthy. On the other hand, Huagu Opera's "Village Officials Are Visitors," portrays a female village chief in leading the villagers to cultural prosperity and so on. These films vividly illustrate ideas and concepts in the new rural development, such as the relationship between cadres and masses, economic and cultural growth, and individual values and pursuits.

Among them, "The Road to a Well-off Society" is a unique work. It uses ancient shadow puppet art to present contemporary stories. The shadow puppetry in the film integrates cartooning, threedimensionality and drama into one, hence, its artistic presentation is fairly innovative. According to historical records, shadow puppetry began in the Western Han Dynasty, thrived in the Tang Dynasty, and flourished in the Qing Dynasty. It has a history of more than two thousand years and is called the "living fossil" of world cultures. In 2011, it was selected in the "Representative List of the 
Intangible Cultural Heritage of Humanity." As an ancient Chinese art, this rare shadow puppet show allows more people to see its artistic forms and appreciate its charms.

These works are based on actual real-life events. They create a distinctive image for the character while conveying the characters' emotions in a modern language context. They also try to fit these events to current ideas and concepts in regard to the story, artistry and performance level.

\subsection{A diversified large-scale production}

Throughout the production of films, the industry has gone through practical explorations, as well as a studio and theater troupe-led approach. In this century, with the collaboration of various creative concepts, the key national cultural project which is the "Peking Opera Film Project," the "Plum Blossom Award Digital Film Project," and the "Rescue Project of National Cultural Repertoires" has taken productions to a new level. The first two projects were sponsored by the China Federation of Literary and Art Circles, the Chinese Theatre Association, and the Central News Documentary Film Studio. Led by the cultural authorities to form planned and large scales production models, the collaborations were effective. Various provinces and cities have also developed "projects" such as the "Rural Film Local Opera Project" in Hunan Province, the "Guangzhou Cantonese Opera Film Boutique Project" in Guangzhou City, and the "Chinese Opera Film Innovation Project" in Henan Province. In these productions, strict artistic standards, advancement plans and reasonable timelines for completion have been devised. In addition to that, exceptional plays from local operas were adapted to the screen, which shows the stage performance of famous operas, subsequently, promoting films and satisfying people's cultural appreciation.

Compared with the previous productions of major operas, this method has allowed local films to be screened at a faster rate. They can also be displayed and disseminated in the form of movies, which forms cultural diversity in production industries.
Production of Chinese opera films during this period is focused on the retention of traditional art, while keeping up with the development and changes, thereby providing possibilities for the formation of new aesthetic concepts.

\section{Evolution of New Aesthetic Concepts and the Formation of Aesthetic Images}

In this century, traditional culture has once again contributed to the development in opera art. In December 2001, the Ministry of Culture devised the "Ten-Year Plan for the Protection and Revitalization of Kunqu Opera." At the same time, the collaboration of the Ministry of Culture and the Ministry of Finance introduced a series of policies such as the "National Stage Art Quality Project Implementation Plan and Project Management," as well as the "Management of Special Funds for the National Fine Stage Art Projects." These plans and projects have ushered in more comprehensive development opportunities for the opera art. In 2014, General Secretary Xi Jinping hosted a symposium in Beijing on literatures and artworks, whereby he clearly stated that the "great rejuvenation of the Chinese nation" required the vibrance in Chinese culture. In July 2015, the national opera symposium was also held in Beijing to further emphasize the significance of revitalization of opera. It also introduced goals and structures for the opera art performing groups, script productions, talent trainings and other aspects. These series of actions reflected the importance of opera in the development of the country and national culture. With this concept in mind and the rapid development of film concepts, it has brought more value and new aesthetic pursuits to Chinese opera films.

\subsection{New aesthetic trend and "new opera- centered" approach}

The merging of the aesthetic beauty of opera with the pursuit of reality in films is an issue in productions for a long time. In previous works, it is based on the "traditional opera-centered" concept. ${ }^{[1]}$ The practice of this concept has formed a "stage documentary" and a "opera art film" with the 
blending of the two. Director Cui Wei proposed that "films must be subjected to the opera" concept. However, there is also the "opera-featured films" concept which leans more strongly to actual films presentations. As the integration of culture and art has become more profound, the issue of virtual and reality has gradually settled. The opera film productions have undergone great changes; from the narratives, to aesthetics, performances, expressions, and the techniques of opera. These new concepts have brought opportunities to the production of opera films and the evolution of aesthetic concepts, whereby the multi-level expression of aesthetic images in opera films can be observed from more than a hundred works. From the beginning, artistic elements in opera have been trying to form connections with modern culture and aesthetics. Then, there was a breakthrough, whereby experimental interests such as the abstract expressions in dialogue, the intervention of the author's subjective viewpoint, etc., promotes the "new opera-centered" approach. ${ }^{[1]}$ This approach refers to the breakthrough changes and developments that have emerged in opera film production ever since this century." "[1] It includes more expressive methods of filming by strengthening the narrative style, visual effects and cinematic techniques, which creates new aesthetic forms and values in order to reflect the producers' expression of opera art. The pursuit of aesthetics has promoted the advancement of filming.

\subsection{Unique aesthetic expressions in experimental works}

In view of new aesthetic trends in this century, several works with unique styles were extremely eye-catching. For example, the "Spring Boudoir Dream" and "The honest official Yu Chenglong."

Director Guo Baochang broke the traditional understanding and aesthetic habits of opera movies first, whereby he filmed the "New Concept Opera Movies" and "Spring Boudoir Dreams". The "New Concept" refers to adopting avant-garde cinematic techniques. This is a new genre created by different formats and advance multimedia technology. ${ }^{[3]}$ Although Guo Baochang's version of "Spring
Boudoir Dream" has made a revolutionary adaptation of the original script, it still conveys respect to the art of opera. He boldly added new story plots and creative elements to the visual effect. In the film production, conceptualization, symbolization, and imagination were used. The film emphasized on a liberal approach while preserving the feeling of a stage in addition of a supplementary feature to illustrate the development of Chinese Peking Opera. The presentation of Duanxian was realistic, and the music was the same as that of an opera performance. In addition to that, Tongari's modern dance was included to the bridal chamber scene, in order to create a refreshing artistic effect. This was a interesting yet, valuable attempt.

In 2008, the director of Zheng Dasheng's “The Honest Official Yu Chenglong" had made new breakthroughs in spatial modeling and visual creation, developing the prospects of Chinese opera films production. The director faced several difficulties while shooting the film. Firstly, the issue of integrating the characteristics of opera into a film, then, solving the issue of merging the virtual and reality aspects, and lastly, portraying both the historical and modern values in its production with a newly written historical-setting script. ${ }^{[4]}$ These three problems were resolved with a new aesthetic effect in his work by exposing the studio itself including the scenery and lights to allow the appreciation of both the realistic and virtual performance as if the audience were watching an opera. ${ }^{[5]}$ At the beginning of the film, $\mathrm{Yu}$ Chenglong walked into the historical scene that is created on stage and at the end of the film, he walked back into the present time and space. On the other hand, music or sounds are common techniques used in films and opera stages. However, among the many works of opera films, the effect this time seemed to be different, as it created both stage-like and cinematic experiences.

These films exude a unique aesthetic charm that is different from the normal opera documentaries, art films and opera-featured films. They give a sense of experimentation and personal expression which are strengths and have profound 
impact on aesthetic expressions.

\subsection{Continual enhancement of the concept and awareness of pursuing the filmization of Chinese traditional opera}

It has always been a topic of discussion to rid the stage-recording styles and to pursue cinematizations to meet the aesthetic needs of modern audiences. Especially after the concept of films and operas were more well-known and accepted by the public, there were views that productions should be more cinematic. As a result, from 2000 to 2015 , there were only a few stagerecorded productions, indicating that the pursuit of opera filmization had been enhanced.

The Peking opera's "Prairie Mother" and "Pair of flower guns" reflected the ethnic minorities. One of it was performed in the natural environment while the other was performed on the stage itself. However, both were filmed and made into dramas. While seeking a balance between the real and virtual, they also emphasize on film-like expressions.

"Prairie Mother" is about prairie herders adopting homeless orphans from Shanghai due to natural disasters in the 1960's. Subsequently, in the narration, these children thrived under tender loving care. It is a challenge to use the art of opera to illustrate the life and emotions of herdsmen. However, a style similar to "The Sound of Music," which is an American film with harmonious singing, involving both the outdoors and indoors setting is terrific. ${ }^{[6]}$ Hence, this opera film formed their own unique way of performance by combining the movements styles of Changtiao, Jinghu, Matouqin, and the Peking Opera with concrete actions as well as "sing along movements."

Although the Peking Opera's "Pair of flower guns" is performed on stage, the film makes full use of the space. The background was extremely simple as it was designed as an abstract geometrical backdrop. A clear performance area was also demarcated on the screen. The lights brightened the performance area, and the characters could freely move in that area. It creates a sense of stage in the virtual form, but in fact, it is a free performance space when viewed in the theatre. During the performance, there was a 24-minutes presentation which utilized digital technology in addition to traditional papercuts, delicate landscape paintings, other imagery emblems and vocal content. This makes the singing visually enjoyable and at the same time, it plays an interpretive role in the characters' image, the director's imagination, narration, and reflections, as well as emphasizing the artistic expressions. ${ }^{[7]}$ In this performance, the addition of technology enhanced the original stage performance, hence, the emotions of the characters were profoundly displayed.

In general, productions in this is period have undergone profound changes, which are reflected in the emphasis on the characters, combination of multi-angles, multi-scenes, long and moving shots in narratives, integrating singings during performances as well as the editing process, forming visual effects between the characters and backgrounds, allowing the entire filming scene to be on screen, using new technologies to enhance limitation of space, and making full use of metaphors or symbols to enhance the artistic charm of opera. In regard to all these changes, the integrity of the performance is still preserved. Whichever methods or techniques, this breakthrough enhances the film's interpretation, lens representation of the opera art, and also new aesthetic concepts in the production of opera films.

\section{Cultural Causes of Continuous Development and the Expansion of Communication Channels 4.1 Cultural causes of continual development}

For more than hundred years, throughout the history of opera films development, its productions has been swinging from sluggish states to the "golden age". There are a few reasons for this.

First, the Chinese opera comes from basic traditional folk culture, however, it also has flexibility. Its' narrations which are formed from basic value systems of moral and ethical concepts reflects the nation's identity. This value system is based on the continuous source of strength that encourages growth after a downpour.

Second, a large number of Chinese operas have 
continuously adjusted according to social changes and the aesthetic needs of the audience throughout the years. Although there were difficulties in adapting and adjustments, a regulatory and restorative system was present. Hence, new directions of social development and forms of artistic expressions can be rapidly adjusted to.

Third, in the long-term creation of Chinese opera films, film workers have gradually grown into creative modes of continual conscious innovation, change, and development. With operas and films, they once again demonstrate their creative passion and perseverance throughout trends and time.

During this period, the realization of films' artistic values, results in a search of more refined expressions and diversified modes of communication in order to enhance creativity in supporting the development of Chinese opera films.

\subsection{Expansion of communication channels}

Relying on government funding and public welfare in opera productions have made slight improvements in the opera films industry. However, it has not significantly advanced in large-scale productions and operations. Although in terms of operations, there are factors that have benefited the film industry such as the appeal of the actors or actresses, the promotions by producers, the quality of films, and the attention of the audiences. For example, two editions of "A Dream of Red Mansions" (Yue Opera) has been successfully launched in the Beijing and Shanghai theaters. At the same time, more than 20,000 sets of DVDs were sold, which achieved good box office results. The question of how Chinese opera films should be disseminated requires continuously emphasis and exploration.

In 2000, the "2131" rural film project has been officially launched. This is a film and cultural enhancement project, whereby at least one film is produced every month in every village. Traditional opera has developed from the folk culture, and its contents reflect the customs and cultural heritage of the folks in a certain area. The establishment of the "2131" project has opened up channels for the broadcasting of Chinese opera films. Chinese opera films have discovered and used broadcasting platforms in the most convenient way. However, on the other hand, there is realization of the need to "return Chinese opera to the people" in order to enrich the spiritual growth and cultural needs of the rural people.

As of $30^{\text {th }}$ June 2015 , the platform has 88 opera films available for orders, of which 86 have been requested by theatres, comprising more than 20 operas, including the Peking Opera, Yu Opera, Huangmei Opera, Huagu Opera, Qin Opera, Yue Opera, and Errenzhuan. A total of more than 3.05 million shows have been ordered for each type of operas whereby the Lv Opera, Yu Opera and Huagu Opera are the most distinctive. In the first half of the year, orders for opera films totaled 624839 , accounting for $10.26 \%$ of the total order. ${ }^{[8]}$ The richer its' cultural content, the more valuable it is. At the same time, improvement of community and digital theatres, pre-screenings, as well as various forms of communication initiated by the rapid development of internet technology provided platforms for the promotion and broadcasting of Chinese opera films.

Undoubtedly, the lifeline of Chinese opera films is closely linked with the value of the opera stage.

\section{Conclusion}

Since the beginning of this century, Chinese opera and films have made considerable progress. In this era of globalization, the context of a diverse world culture increases the importance of protecting and developing national cultures. Development of intangible cultural heritages and the inheritance of these cultures have significant value made in reference to the aesthetic psychology of the modern society. Taking all these into consideration, there are needs in the Chinese opera films industry. First, renewing concepts, using new technologies and modern means of communication are necessary methods for the development of opera films. Second, the balance between "Chinese opera films" and "Chinese film opera" should be practiced. Third, it is necessary to ensure opera films have a 
more distinctive sense of the era and its modernism. Hence, with all these, the charm of the opera art can be better emphasized. There are needs for continuous exploration to improve and develop Chinese opera films in hope that the industry will once again usher in a new "golden age."

\section{Disclosure statement}

No conflicts of interest.

\section{References}

[1] Gao, X(2015). On the Art of Traditional Chinese Opera. China Film Publishing House, p. 299.

[2] Cui, W(1982). The Experience of Shooting Chinese Opera Films in Cui Wei's Art World. China Film Publishing House, p. 86.

[3] Guo, B(2004). Is Nobody Watching It or Is It Extremely Popular, Spring Boudoir Dream Plays a New Concept. http://www.chinanews.com/news/ 2004/2004-12-10/26/515217.shtml

[4] Nie, W., Guo, L(2009). The Intertextuality of "Shadow" and "Play" and Modernity Innovation in Narrative - Minutes of the Symposium on the Peking Opera Film "The Honest Officials of Yu Chenglong". New Films, Issue 5.

[5] Li, J(2012). "Opera Film: At the Junction of Opera, Film and Politics - A Summary of the International Academic Symposium on Opera Film", in "The Art of Chinese Opera". Issue 8.

[6] Yu, L(2006). In an interview with Yang Xiufeng, a teacher of the Directing Department of the Chinese Academy of Traditional Chinese Opera in Beijing on May 16.

[7]Zhang, J(2006). "The Flower Spear: Digital Technology Reshapes Classic Chinese Opera Movies." China Film News.

[8] (2015). "Inventory of the Mobile Film Market in the First Half of 2015". Film Digital Center. http://www.dmcc.org.cn/mainSite/xw/zxgz/71224 7/index.html 